\title{
Microscopic investigations into lubricated friction pairs in synchronizers
}

\author{
G Poll ${ }^{*}$, S Neudörfer, and T Skubacz \\ Department of Machine Elements, Engineering Design and Tribology, Leibniz University Hannover, Hannover, Germany
}

The manuscript was received on 10 September 2007 and was accepted after revision for publication on 28 January 2008.

DOI: 10.1243/13506501JET353

\begin{abstract}
An essential component of manually operated vehicle transmissions is the synchronising device. Synchronizers have the task of minimizing the speed difference between the shifted gearwheel and the shaft by means of frictional torque before engaging the gear. Proper operation requires a sufficiently high coefficient of friction. An optimized design of the system with regard to appropriate function and durability on the one hand as well as low cost, low mass, and compact over-all dimensions on the other hand today requires time-consuming and expensive component testing. As it is easier to coat plane surfaces with a friction material than to coat the inner or outer cones of synchronizer rings, this paper investigates if the component tests can be substituted by simpler pin-on-disc type model tests. For this purpose, such model tests and tests with original synchronizers are compared, in particular, regarding the friction characteristics of different materials. Furthermore, a fluorescence-based method is successfully applied to observe the lubricant distribution and the contact conditions during shifts. The investigations reveal that there are limitations to the applicability of tests with plane model pins to real conical component behaviour, which can partly be attributed to differences in drainage efficiency.
\end{abstract}

Keywords: synchronizer, synchromesh, gear, pin, pin-on-disc, disc, cone, friction, clutch, lubricant, fluorescence, thickness of lubricant, distribution of lubricant, micro-contact

\section{INTRODUCTION}

One important component of manually operated vehicle transmissions is the synchronising system. Its task is to reduce the speed difference between the shaft and the idler gear during the gear shift by means of friction before positive engagement. To ensure proper functioning, a sufficiently high coefficient of friction is essential. In addition to the geometry and material properties of the synchronizer friction pair as well as lubricant properties, tribological stress conditions also have an influence on its operation.

Today, extensive and time-consuming component tests with expensive test parts are conducted to develop friction materials for synchronizers. As it seems easier to coat plane surfaces with a friction

\footnotetext{
*Corresponding author: Department of Machine Elements, Engineering Design and Tribology, Leibniz University Hannover, Welfengarten 1A, Hannover 30167, Germany. email: poll@imkt.uni-hannover.de
}

material, this paper investigates if the component tests can be substituted by simpler pin-on-disc type model tests. Therefore, the idea is to compare pin-on-disc type model tests and tests with real synchronizers regarding different aspects, i.e. friction characteristic and contact conditions.

\section{OPERATION OF A SYNCHROMESH}

Figure 1 shows the components of a synchronising system in an exploded view. The cone is positively engaged with the gear. The synchro body, the synchro sleeve, and the synchro ring as well as the springs and the centering pieces of the pre-synchromesh unit are the components that rotate with the shaft. The synchro sleeve can be displaced axially during gear shift with the aid of the gearshift linkage in the direction of the gear. This presses the ring against the cone via the stop teeth. The difference in speed is reduced by friction between the conical friction surface of the ring and the cone. 


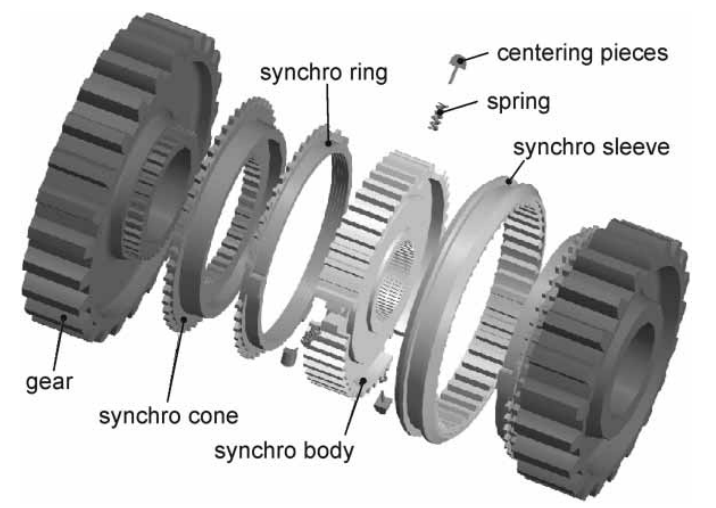

Fig. 1 Structure of a self-locking synchronizer system

When the speeds have been equalized, positive engagement is created by shifting the synchro sleeve teeth into the spaces between the clutch teeth. Proper operation is only ensured if the frictional torque in the conical surface between the ring and the cone is sufficiently large. The frictional torque arises corresponding to the coefficient of friction by axially pressing the ring against the cone (Fig. 2). The normal force in the contact results from the axial pressing force intensified by the cone angle. The coefficient of friction in the conical surface may change due to altered tribological conditions.

An additional function of synchromesh gears is that they are intended to prevent positive engagement until speeds have been completely equalized. For this to happen, the locking condition must be fulfilled. The frictional torque, $T_{R}$, must be greater than a reverse torque, $T_{Z}$, which occurs as a result of the pitch angle at the teeth faces. In this case, the opposing teeth prevent further shifting of the synchro sleeve. The locking condition gets violated by decrease in the coefficient of friction and axial wear (pressure is no longer intensified by the cone angle). In addition, pitch wear causes a reduction of the pitch angle which may, due to the rising aligning torque, $T_{Z}$, result in violation of the locking condition. Any malfunction in the system makes a noticeable grating sound when the teeth of the sliding

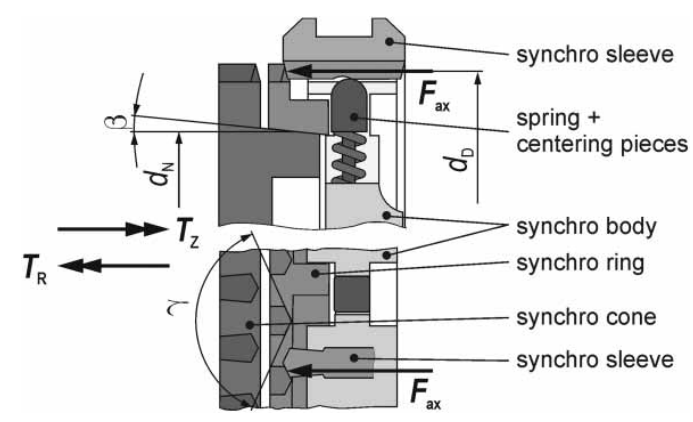

Fig. 2 Operation of a synchromesh gear sleeve and the cone meet whenever there is a speed difference.

\section{EXPERIMENTAL METHODS}

\subsection{Test rig}

The experiments were conducted on a special test rig (Fig. 3). The basic principle of the test rig is that the test rig shaft is coupled with a flywheel and accelerated by an electric motor to a predetermined speed. Then the shaft is braked by pressing the friction surface against the stationary measuring device.

The gear is shifted by means of a hydraulic cylinder. The force of the hydraulic cylinder is controlled in order to achieve a defined build-up of load. The system is lubricated by a temperature-controlled oil circulation. The test specimens are connected to the test-rig shaft and to a force-sensing device.

An inductive displacement sensor measures the axial displacement of the ring with regard to the cone. When the axial force is constant, the displacement corresponds to the wear in the friction surfaces. The sensor device, specially developed for this test rig, measures axial force and frictional torque directly at the test specimen, so that it is possible to precisely determine the coefficient of friction in the friction surfaces. The computer-based control system dynamically records the reaction force and torque, the wear and the component temperature for each single shift.

\subsection{Set-ups for component tests and model tests}

There are many outside parameters e.g. wear on the locking teeth beyond the friction pair itself that influence the complex real synchronizer systems. For that reason, the system is reduced to a simple single-cone clutch, in the following referred to as 'component test'. With a special system of adapters, also pin-on-disc type 'model' tests can be run on the same test rig. Figure 4 describes both test set-ups.

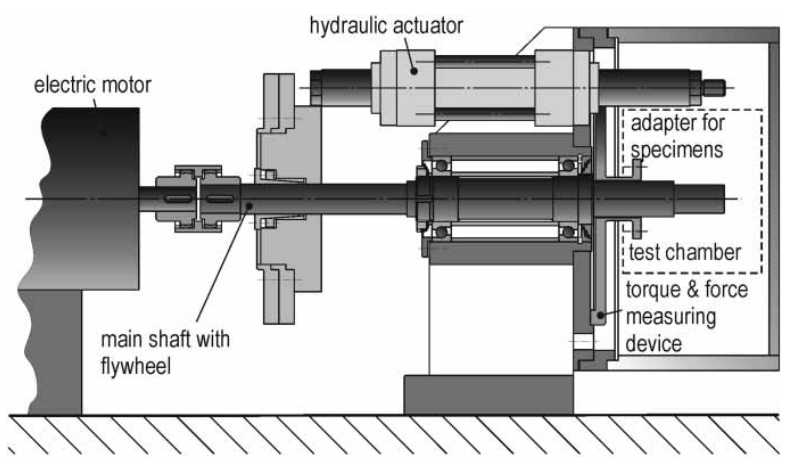

Fig. 3 Side-view of the friction surface test rig 

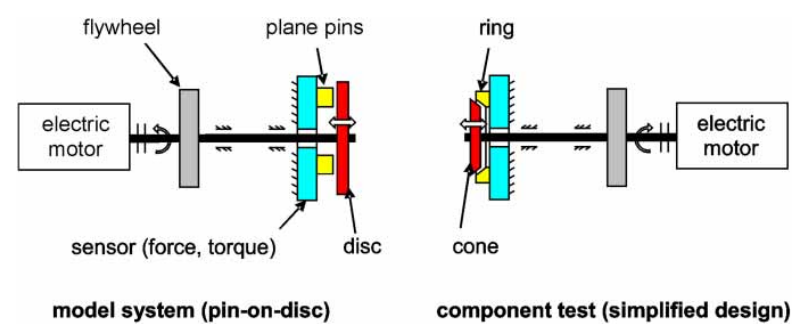

Fig. 4 Test set-up for component and model tests

To ensure that component tests (index $K$ ) are comparable to model tests (index $P$ ) using plane pin-ondisc type contact it is necessary to apply the same stress to both friction systems. Therefore the average nominal pressure, $p_{m}(1)$, the initial sliding speed, $\mathrm{v}_{\max }$ (2), and the sliding time, $t_{r}$ (3), ought to be equal.

Average nominal pressure

$$
p_{m, K}=\frac{F_{a x, K}}{A_{K} \sin \alpha} \stackrel{!}{=} \frac{F_{a x, P}}{A_{P}}=p_{m, P}
$$

Initial sliding speed

$$
\mathrm{v}_{\max , K}=\omega_{K} \bar{r}_{K} \stackrel{!}{=} \omega_{P} \bar{r}_{P}=\mathrm{v}_{\max , P}
$$

Sliding time

$$
t_{r, K}=\frac{J_{K} \omega_{K}}{T_{R, K}} \stackrel{!}{=} \frac{J_{P} \omega_{P}}{T_{R, P}}=t_{r, P}
$$

The friction torque is approximately calculated as

$$
T_{R, K, P}=\mu_{K, P} \underbrace{p_{K, P} A_{K, P}}_{F_{N, K, P}} \bar{r}_{K, P}
$$

with $\bar{r}_{K, P}$ as medium radius of friction.

Assuming equal friction coefficients, the friction area, $A_{K, P}$, the average friction radius, $\bar{r}_{K, P}$, and the mass moment of inertia, $J_{K, P}$, have to be chosen in a way that equal specific work of friction per shift is reached to ensure similar temperature conditions in the contact. Therefore, the following equation (5) has to be fulfilled

$$
\frac{p_{m, P}}{p_{m, K}} \frac{\mu_{P} A_{P} \bar{r}_{P}^{2}}{\mu_{K} A_{K} \bar{r}_{K}^{2}} \frac{\mathrm{v}_{\max , K}}{\mathrm{v}_{\max , K}} \frac{J_{K}}{J_{P}} \stackrel{!}{=} 1
$$

Table 1 presents the important parameters of the specimen.

\subsection{Investigated friction pairs}

The experimental investigations were conducted on four tribological systems with friction pairs currently used in synchromesh (Table 2). One system (A) is made of massive brass with a conical thread with trapezoidal grooves. The second system (B) consists of sintered brass material fixed by welding spots and featured with axial grooves. The other two pads named 'carbon fibres 1, 2' (C1, C2) consist of resin-linked fibres and some hard material in different proportions. One of them (C1) features axial oil grooves whereas the other (C2) has none. As the plane pins should be as simple as possible, they are not grooved at all except the massive brass pins.

The steel cones and discs are made of $17 \mathrm{CrNi}-$ Mo6 quenched and tempered to 59HRC before being ground. As lubricant, a standard gear oil BOT 130M $\left(v_{40}=77.5 \mathrm{~mm}^{2} / \mathrm{s} ; v_{100}=15.1 \mathrm{~mm}^{2} / \mathrm{s}\right)$ is applied.

\subsection{Fluorescence-based observation method}

Synchronizers operate in the mixed friction regime, which means the friction behaviour depends on the

\begin{tabular}{|c|c|c|}
\hline & Cone & Pin-on-disc \\
\hline $\begin{array}{l}\text { Medium diameter of friction }(\mathrm{mm}) \\
\text { Contact width }(\mathrm{mm}) \\
\text { Area of friction surface }\left(\mathrm{mm}^{2}\right) \\
\text { Cone angle } \\
\text { Mass moment of inertia }\left(\mathrm{kg} \mathrm{m}^{2}\right) \\
\text { Surface roughness }\end{array}$ & $\begin{array}{l}2 \times r_{\mathrm{K}}=76 \\
8 \text { (ring width) } \\
A_{\mathrm{K}}=1910 \\
2 \alpha=13^{\circ} \\
J_{\mathrm{K}}=0.273 \\
\text { Steel: Ra } 0.1 \ldots 0.2 \mu \mathrm{m} \\
\text { Sapphire: optical polished }\end{array}$ & $\begin{array}{l}2 \times r_{\mathrm{P}}=105 \\
20 \text { (pin diameter }) \\
A_{\mathrm{P}}=942 \\
-\left(180^{\circ}\right) \\
J_{\mathrm{P}}=0.267\end{array}$ \\
\hline
\end{tabular}
proportion of solid-solid contact and lubricated contact. Lubricant filled cavities are situated between the single solid-solid contact spots. To be able to

Table 1 System parameters 
Table 2 Investigated friction pairs (arrow in circumference direction)

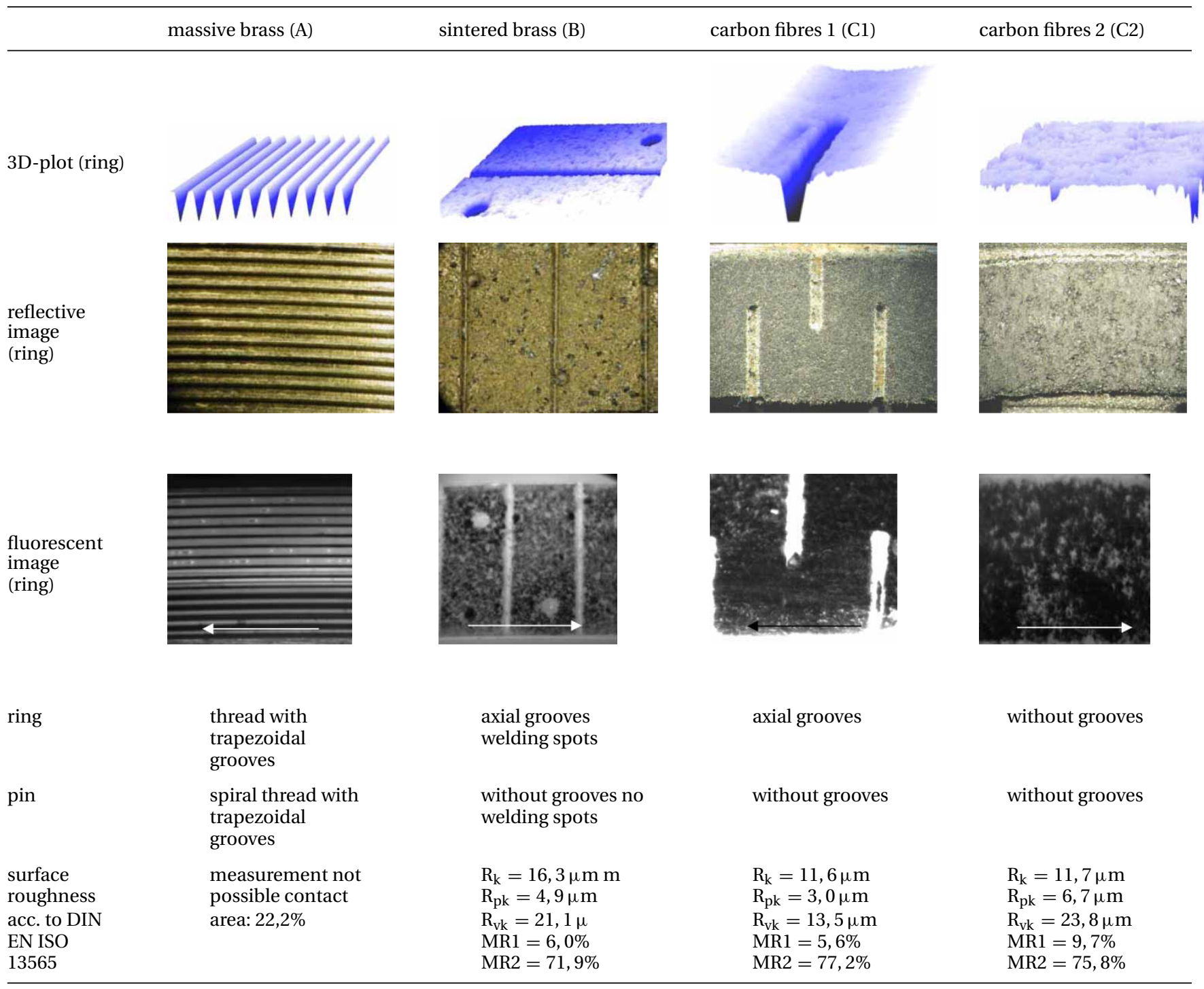

identify these different zones, two main tasks have to be fulfilled:

(a) optical access into the contact zone has to be provided;

(b) oil needs to be visualized.

The former is achieved through substituting the steel surface by a transparent sapphire counterface. Its physical properties closely resemble those of steel. In this way, especially the thermal boundary conditions will be similar. The second problem, visualising the oil, can be solved by exploiting the natural fluorescence described in the following.

Fluorescence is the spontaneous emission of light after previous excitation with radiation of higher frequency. Here, the light source is an UV-flash. Since in this case, the natural fluorescent response of the pure oil was intense enough, no additional fluorescent dye needed to be added in the first instance. The excited lubricant behind the sapphire counterface emits light in the visible spectrum, which can be filtered and recorded by a microscope-camera layout (Fig. 5).

The intensity of the emitted light depends on the thickness of lubricant film as shown in Fig. 6. Because

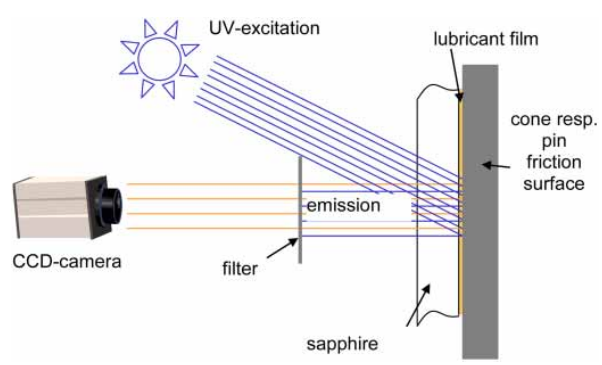

Fig. 5 Principle of fluorescence-based observation method 


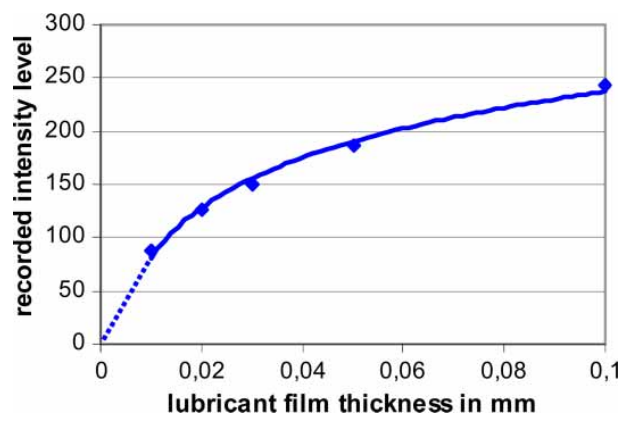

Fig. 6 Calibration curve fluorescence intensity versus lubricant film thickness

of the distinct topography of the friction surfaces, it is not possible to work in the dashed linear range. There is immediately available evidence about the distribution of lubricant, as bright areas in the pictures are indications for a local high lubricant film thickness.

\section{EXPERIMENTAL RESULTS}

\subsection{Friction characteristics}

Exemplarily, measurements with an initial sliding speed of $1 \mathrm{~m} / \mathrm{s}$ and a nominal pressure of $1 \mathrm{~N} / \mathrm{mm}^{2}$ are represented here to compare the model plane pin on disc contacts with the conical original component contacts. Due to the low loading, the wear rate is nearly zero (the axial wear is below $0.01-0.04 \mathrm{~mm}$ over 100.000 cycles). Figure 7 shows characteristic trends of friction, axial force, and speed during the course of single shifts.

For the massive brass systems (A), the friction behaviour of both geometries matches fairly well whereby the friction coefficient rises moderately towards the end of the shift. In contrast, the friction trends of sintered brass (B) and carbon fibres (C1) differ in multiple aspects.

Taking a closer look at a single shift with friction pair $B$ reveals that the friction coefficient of the plane model system starts lower than the one of the conical original system, but then increases faster and finally reaches the level of the conical system at the end of the shift.

The conical system $\mathrm{C} 1$ is generally characterized by a decrease in friction coefficient with decreasing sliding speed towards the end of the shift. For the plane model contact, the level of friction is much lower at the beginning and then approaches the friction coefficient of the original system but remains slightly lower throughout the remaining time of the shift.

Furthermore, an influence of the direction of rotation was detected, exemplarily shown for system A in Fig. 8.

\subsection{Micro contacts and lubricant distribution}

The following section shows exemplary fluorescence images of the lubricant distribution and the micro contacts in the contact area.

Figure 9 shows fluorescence images of the conical sintered brass system B with oil filled axial oil grooves

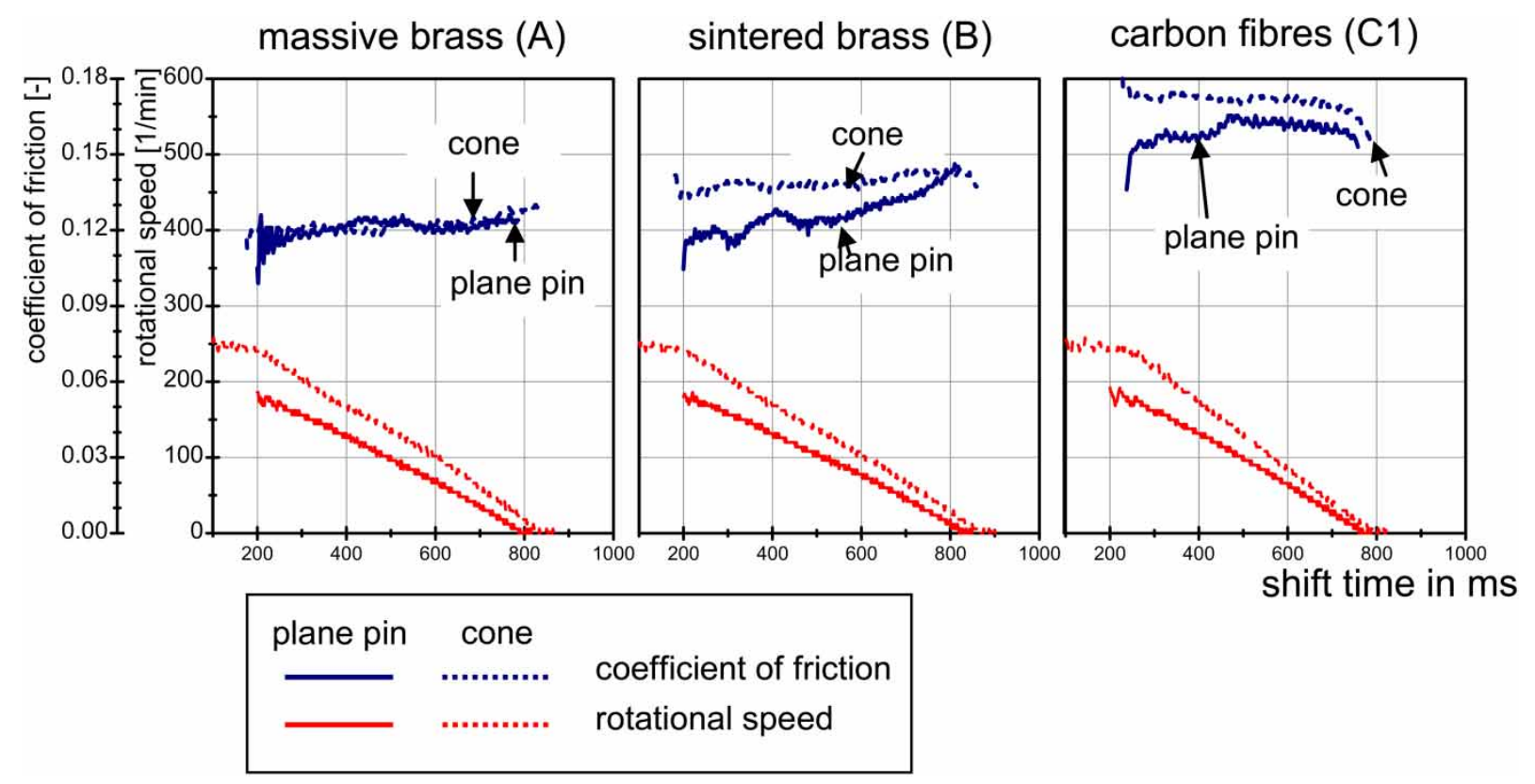

Fig. 7 Comparison single shifts of plane model and conical component tests of A (left), B (middle), and Cl (right) 


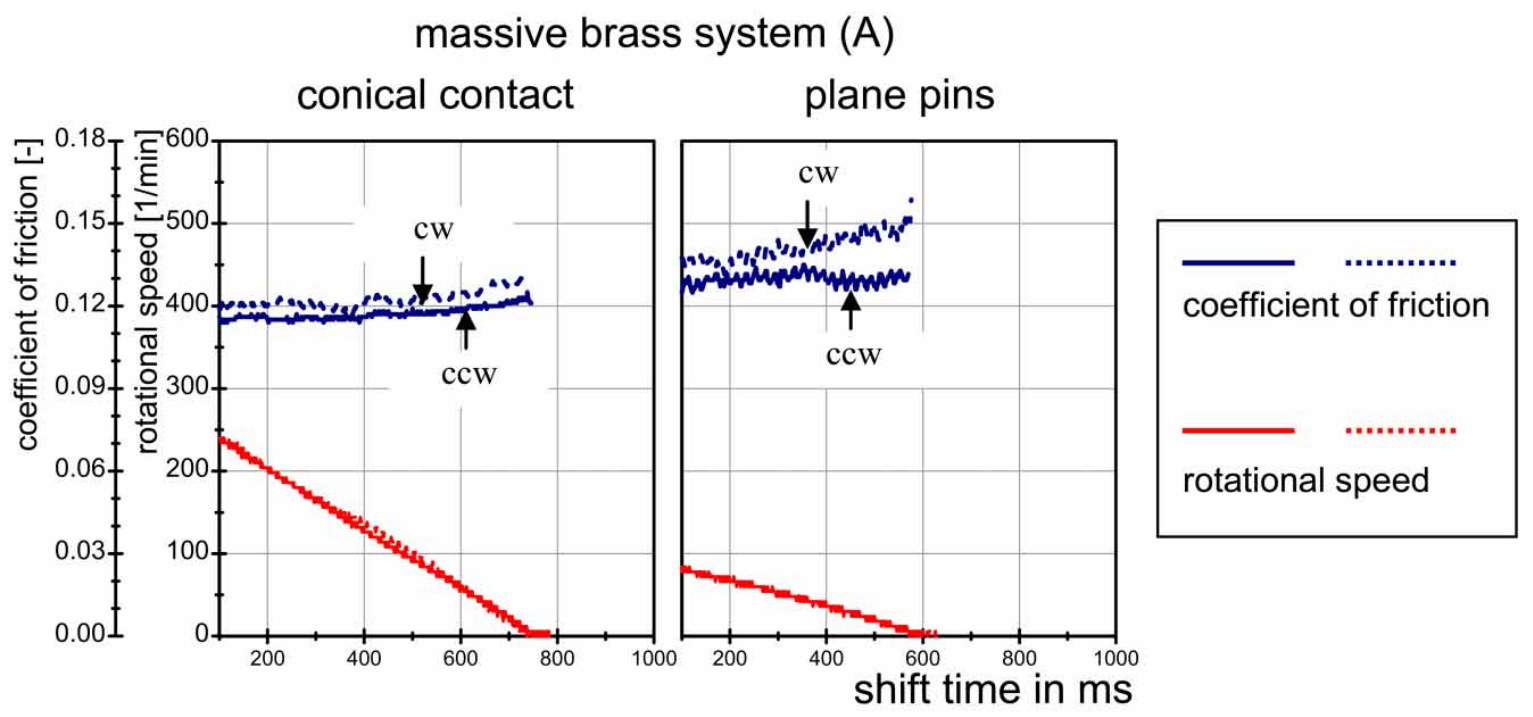

Fig. 8 Single shift graphs for system A at different directions of rotation

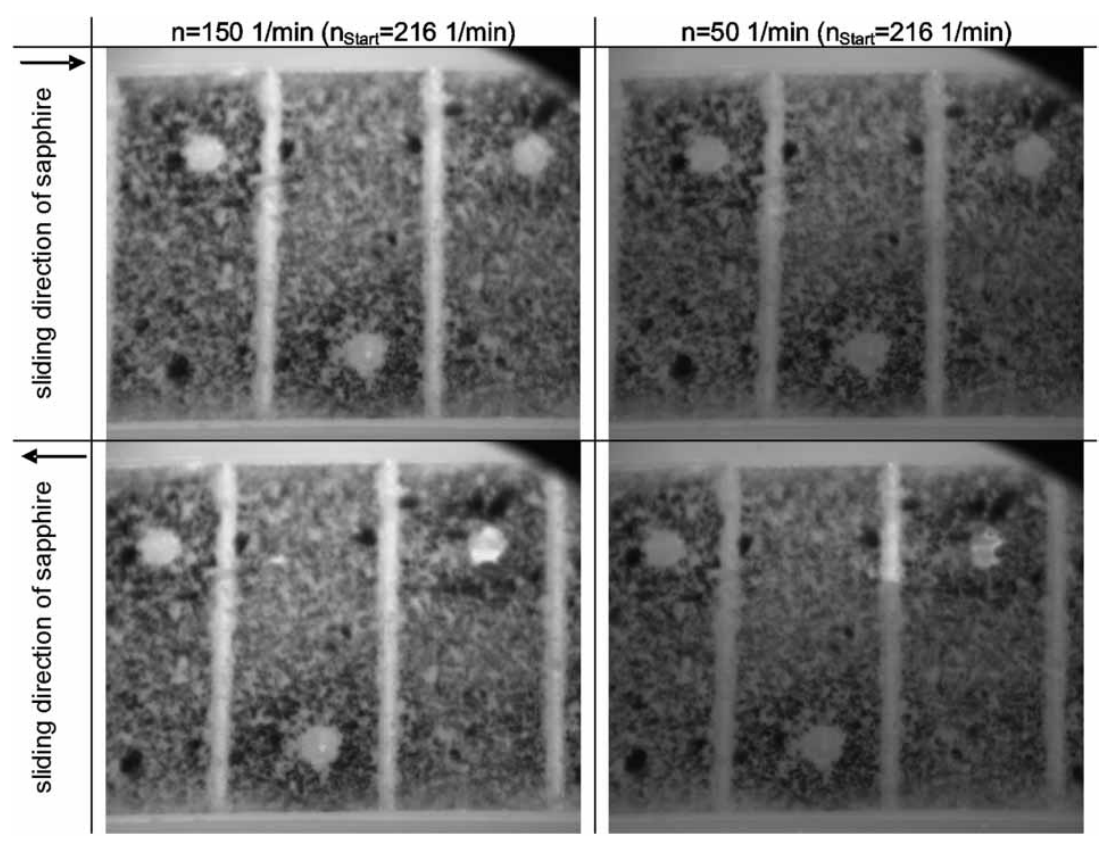

Fig. 9 Fluorescence image B conical

and welding spots. Around the welding spots, a higher contact density can be recognized. Reversing the direction of rotations alters the lubricant distribution and hence fluorescence intensities especially in the welding spots and the drainage grooves (comparison upper and lower row of images). This effect could be due to the local macro and microstructure of the surface. Both rows of images indicate that the lubricant film thickness decreases towards the end of the shift.

The corresponding plane system B is shown in Fig. 10 at the end of a shift for different rotational directions, resulting in clearly visible differences in lubricant distribution. The lubricant piles up in front of the entry into the contact. Behind the contact, the oil is stretched out to streaks on the sapphire. When passing the contact, the lubricant film decreases and splits into single lubricant-filled channels formed by the surface roughness pattern (magnified detail in Fig. 11(a)). Further magnification (Fig. 11(b)) gives an impression about the micro contact situation. The roughness contacts, cavities filled with lubricant and even gas bubbles can be identified.

The plane carbon fibre system C2 behaves in a similar way (Fig. 12). Compared to the perimeter, the contact density appears to be lower in the centre of the 


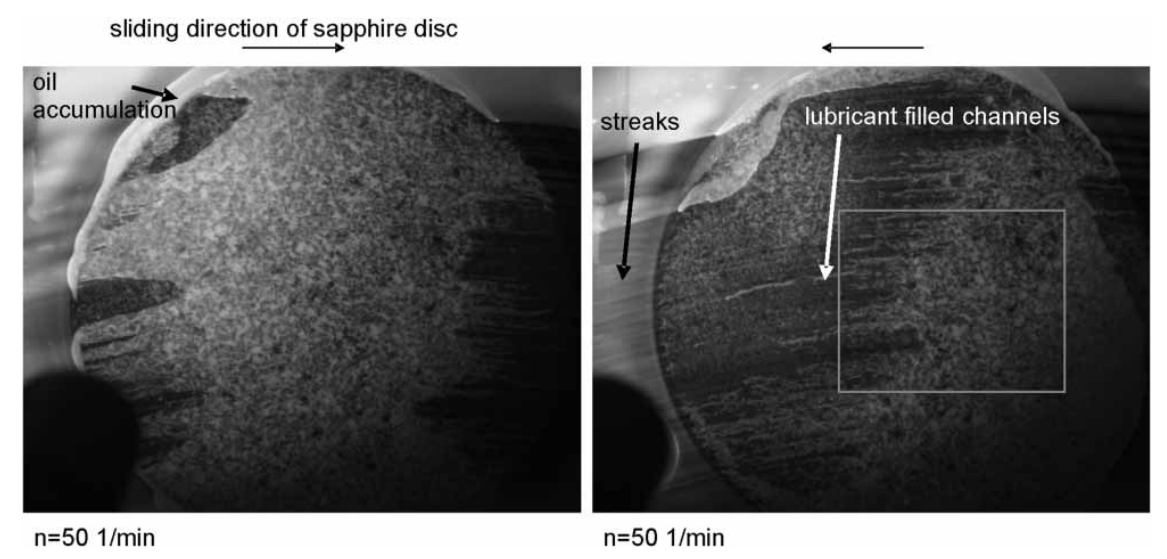

Fig. 10 Fluorescence image B plane

a) zoom I

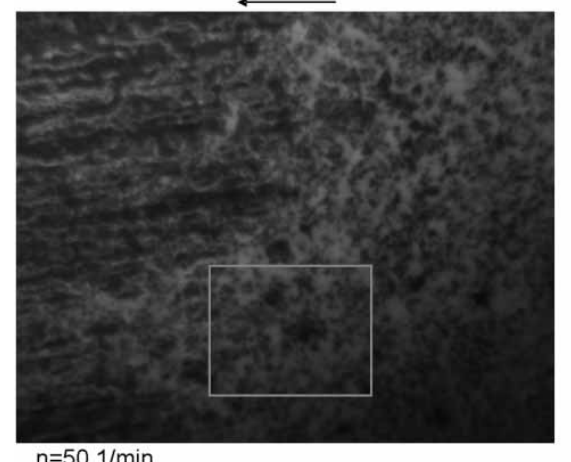

$\mathrm{n}=50 \quad 1 / \mathrm{min}$ b) zoom II

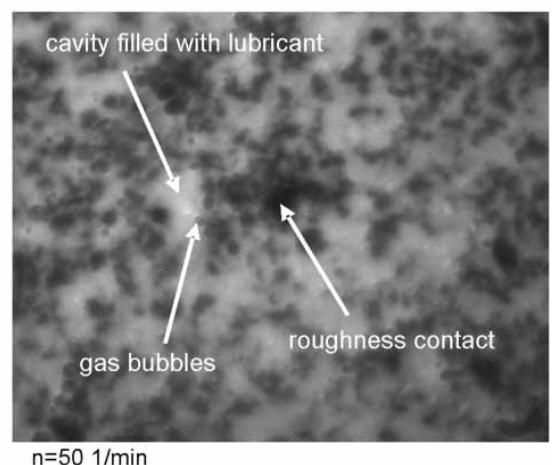

$\mathrm{n}=50 \quad 1 / \mathrm{min}$

Fig. 11 Detail fluorescence image B
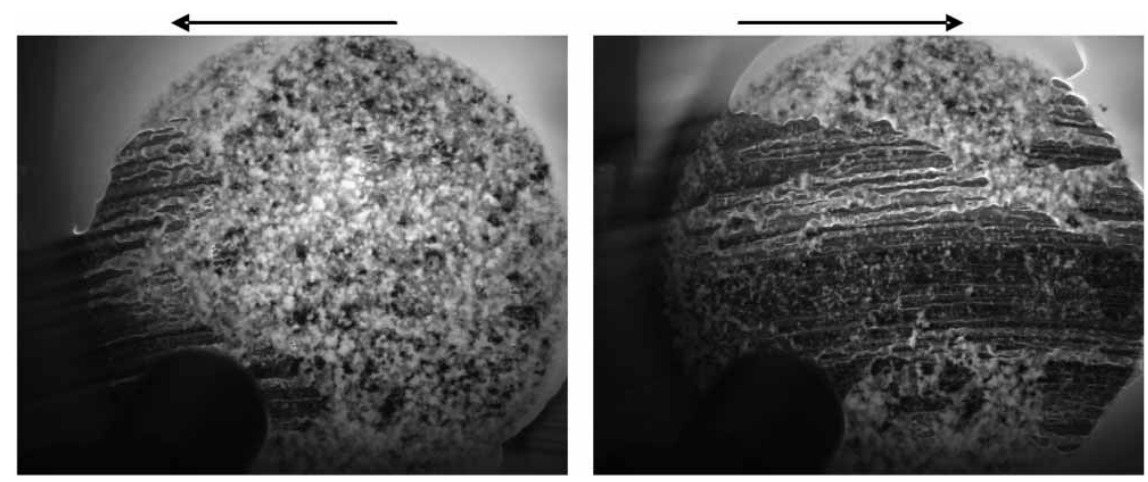

Fig. 12 Fluorescence image C2 plane

pin than in the outer zones. In the opposite direction of motion, the contact is almost entirely stripped from oil.

\subsection{Discussion of the experimental results}

For the investigated friction pairs, the mode of lubrication varies during the shifts within the wide range of mixed friction. Over the course of shifts, the proportion between fluid and dry friction changes to a higher contribution of dry friction. The rising number of roughness contacts leads to an increase in friction coefficient. This transition to dry friction will be accelerated by more efficient drainage features, which help to squeeze the lubricant out of the contact area.

As a consequence, there is a similar behaviour of massive brass systems with plane and conical frictional contacts whereas for the other investigated systems the plane and the conical contacts 
differ considerably. These differences result from two superimposed effects.

1. The width of the rings (conical contact) is smaller $(8 \mathrm{~mm})$ than the diameter $(20 \mathrm{~mm})$ of the plane pins. Furthermore, in order to facilitate manufacturing of the specimen, the plane frictional contacts of $\mathrm{B}, \mathrm{C} 1$, and $\mathrm{C} 2$ have no drainage grooving. Because of that, in plane system, the lubricant has a longer travelling distance when squeezed out of the contact and therefore more time is needed to reduce the proportion of fluid friction. On the other hand, the contacts of the massive brass system have an optimum drainage through the axial and tangential grooving.

2. Achieving complanarity of pin and disc surfaces turned out to be a more demanding task than anticipated. The pin surfaces of the brass system A were finished when firmly attached to the adapter resulting in almost perfect matching of the surfaces. The other friction materials were bonded onto the plane pins without further machining making deviations from parallel alignment and equable pressure distributions more likely.

\section{CONCLUSIONS}

The presented fluorescence-based observation method allows the analysis of lubricant and micro contact distribution in the contact area. The system is applicable both to get overviews and detail images at a certain instant during the shifting process. The images can be used to relate the friction behaviour to the surface microstructure.

The investigations revealed that there are limitations to the applicability of tests with plane model pins to real conical component performance. This can partly be attributed to differences in drainage efficiency.
Furthermore, differences in the properties of the friction layers formed during operation are currently studied by means of mass-spectroscopy and microscratch tests. The results will be presented in the near future.

\section{ACKNOWLEDGEMENT}

The investigations were performed in context of the research project 'Oberflächenbetrachtung zur Reibwertoptimierung von Beschichtungen'. The authors thank Stiftung Industrieforschung (Foundation for Industrial Research, Cologne, Germany) for sponsoring this project.

\section{APPENDIX}

\section{Notation}

A friction area

$F_{\mathrm{ax}} \quad$ axial force

$F_{\mathrm{N}} \quad$ normal force

$J \quad$ mass moment of inertia

$\mathrm{K} \quad$ conical (component system)

$p_{m} \quad$ nominal pressure

P plane (model system)

$\bar{r} \quad$ medium radius of friction

$t_{r} \quad$ sliding time

$T_{R} \quad$ frictional torque

$T_{Z} \quad$ restoring torque

$\mathrm{v}_{\max } \quad$ initial sliding speed

$\alpha \quad$ cone angle

$\mu \quad$ coefficient of friction

$v_{40} \quad$ viscosity at $40^{\circ} \mathrm{C}$

$v_{100} \quad$ viscosity at $100^{\circ} \mathrm{C}$

$\omega \quad$ angular velocity 